\title{
Pothole Detection System
}

\author{
Tushar Premanand, Sriram Sridhar, Abhinav Durbha
}

\begin{abstract}
Potholes are a common nuisance that most people have had the displeasure of coming across. These bowl-shaped cavities in the road cause a large proportion of automobile related accidents, either directly or indirectly. Begetting the process of getting a pothole covered/fixed is a time consuming one that involves informing the appropriate authorities and having them take action. Implementing a system that involves citizens in the process of detecting pothole is what is being envisioned. The system includes a mobile application which is capable of taking a photo, this photo is then sent to a backend server where it is processed by a neural network model capable of detecting potholes. If any potholes are detected in the photo then the photo is saved along with its geolocation and forwarded to the appropriate authorities thus providing an efficient method of identifying potholes and removing the communication gap between the government and its citizens.
\end{abstract}

Keywords: Accidents, Neural Network, Potholes

\section{INTRODUCTION}

Potholes are depressions which exist on the surface of a pavement that usually consist of a bowl-shape. Potholes are formed when water, from heavy rain or other sources, seep into the underlying soil of the pavement and with repeated pressure from overhead traffic causes a depression to form on the pavement surface. The occurrence of potholes causes a great deal of inconvenience for pedestrians as well as motorists, not to mention a large number of accidents are caused due to potholes. According to statistics released by the Ministry of Road Transport and Highways, over 9300 deaths and 25000 injuries have been caused by potholes over the last few years. Generally, the most common cause of potholes is fatigue. Repeated cycles of stress, such as continuous passage of vehicle wheels over the asphalt, causes the bonds between asphalt and stones to break and the continuous asphalt later to separate in blocks. Conditions that accelerate the speed at which asphalt wears are numerous such as passage of heavy vehicles, intense traffic, bad drainage. The problem can even arise internally from when the road is first built i.e. poor quality of asphalt, insufficient or uneven compacting of the asphalt layers. Being able to accurately identify potholes is of

Revised Manuscript Received on July 10, 2020.

* Correspondence Author

Tushar Premanand*, Department of Computer Science and Engineering, SRM IST Ramapuram, Chennai, India. E-mail: tusharpremanand@gmail.com

Sriram Sridhar, Department of Computer Science and Engineering, SRM IST Ramapuram, Chennai, India. E-mail: sriramsridhar26@gmail.com

Abhinav Durbha, Department of Computer Science and Engineering, SRM IST Ramapuram, Chennai, India. E-mail: abhinavdurbha@gmail.com

(C) The Authors. Published by Blue Eyes Intelligence Engineering and Sciences Publication (BEIESP). This is an open access article under the CC BY-NC-ND license (http://creativecommons.org/licenses/by-nc-nd/4.0/) utmost importance to prevent further accidents. However, the system of manually identifying and assessing is costly and arduous. Therefore, an autonomous system which is capable of identifying and assessing potholes is in high demand. Furthermore, a system that takes the help of citizens in identifying potholes is an efficient method as well. A mobile application is developed that allows citizens to capture photos of potholes and then send to a backend server which is equipped with a neural network model capable of detecting potholes present in the image. The neural network makes use of a model called YOLO v3 which is a new approach to object detection. The unified architecture present in YOLO v3 is extremely fast and processes images at 45 frames per second in real-time. The model is simple to construct and can be directly trained on full images. So, this neural network model has been trained with 1500 images of potholes and is capable of detecting potholes from an image. Once the potholes are detected, the image is saved along with its geolocation and then the image is forwarded to the appropriate authorities thus reducing the gap between the government and its citizens.

\section{RELATED WORKS}

[1] This paper uses a mobile sensing system for road irregularity detection using android OS smart phones. Using selected data processing algorithms, potholes are detected. [2] Uses a blackbox camera to collect data in the form of images and detects potholes using the proprietary algorithm and convey the information to road authorities in the city of Seoul, Korea.

[3] Uses piezo-electric sensor to convert pressure or force into electrical voltage based on GPS module readings on the of the potholes and sends a notification to the user about the potholes coming up ahead through a mobile application.

[4] Uses disparity mapping with computer vision to find out the difference between damaged and undamaged roads and road surface modelling to improve the detection of potholes. [5] Uses vibration meter and accelerometer along with gps module to detect potholes and inform to the authorities so that proper road conditions can be restored.

\section{SYSTEM ARCHITECTURE}

\section{A. PROPOSED SYSTEM}

The first step of the system involves data collections consisting of image and pothole details which is used to train the neural network model. Next stage involves the classifier model which takes either a video or an image as an input. If the image contains a pothole, then the pothole is detected and shown. In the event of it being a video, the video is broken into frames.

\section{Published By:}

Blue Eyes Intelligence Engineering

\& Sciences Publication

DOI: 10.35940/ijeat.F1281.089620

Journal Website: www.ijeat.org

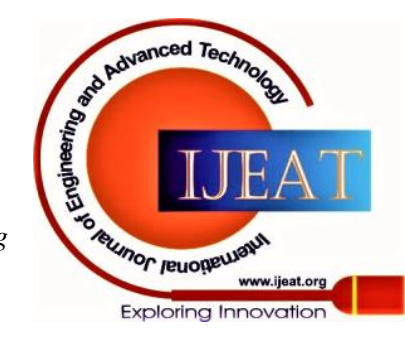




\section{Pothole Detection System}

The frame goes through the same process and if a pothole is present, then it is shown. This way, a system capable of detecting potholes from images submitted by users is implemented.

\section{B. BLOCK DIAGRAM}

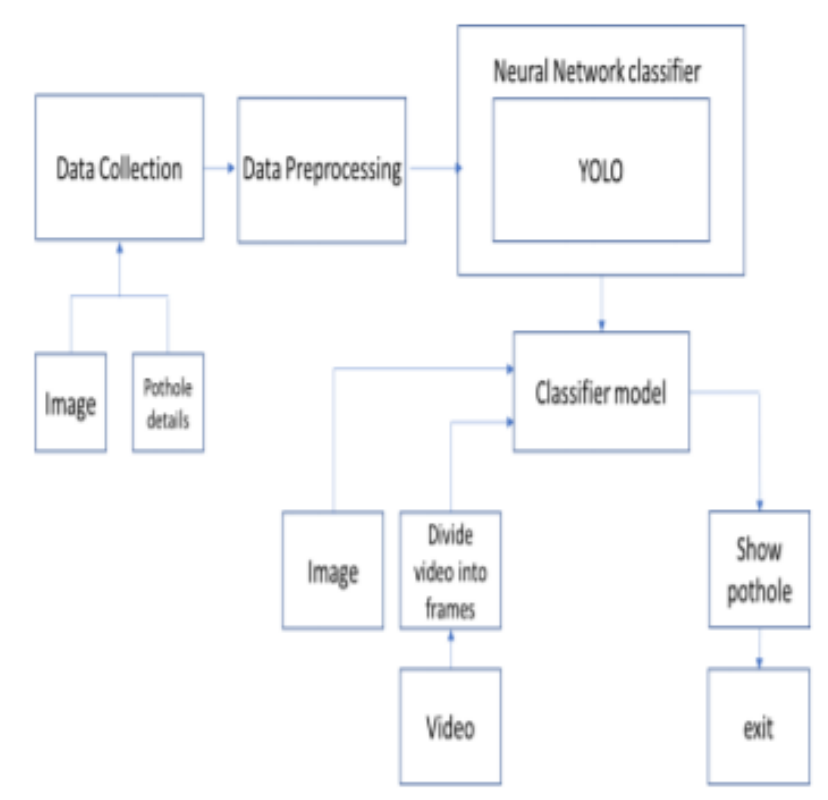

Fig. 1: Block Diagram of Proposed System

\section{DETAILS OF THE MODULES}

\section{A. YOLO}

In YOLO, a different approach is used implementing various networks which make use of a region proposal or a sliding window. Object detection is reframed as a singular problem of regression. Having looked just once at the input image, YOLO divides a grid of cells (S x S) out of it. Every cell predicts a number of bounding boxes (B) along with a score of confidence which constitutes the intersection over union (IOU) and the ground truth bounding box.

\section{B. UNIFIED DETECTION}

Here, each of the object detection components are grouped into a single neural network. Each bounding box is predicted through the network by using features from the whole image. Similarly, every bounding box constituting every class for an image are also predicted simultaneously. Features like real-time speeds and ended training along with high average precision maintenance are seen in the YOLO design. Considering that when an input image is interpolated it is cleaved by the system into a S x S grid, if the center of any object lies on the grid cell, then that particular grid is in charge of object detection. Each cell is responsible for prediction of confidence scores and bounding boxes (B). The reliance of the model based on accommodating an object and its precision of predicting the box is reflected by the confidence scores. Confidence is defined as Prediction (Object)*IOUTruthPrediction. If a cell does not contain an object, the corresponding scores of confidence amount to zero. Otherwise, the scores are equal to the IOU between the ground truth and the predicted box. Every bounding box constitutes of five predictions: w,h,x,y and confidence. The height (h) and width (w) are predicted in relation to the entire image. The center of the box in relation to the grid cell bounds are represented by coordinates $(\mathrm{x}, \mathrm{y})$. The confidence prediction is the representation of the IOU between any ground truth box and the predicted box. Every grid cell is also used for predicting conditional class probabilities (C), Prediction (Class i|Object). Each and every probability is conditioned on the object containing grid cell. Despite the number of boxes (B), only a single set of class probabilities are predicted per grid cell. During time of test, the individual box confidence predictions and the conditional class probabilities are multiplied resulting in the class specific confidence scores for every box. Both the probability of appearance of class in box and how well the box predicted fits the object are encoded in the scores. Prediction (Class $\mathrm{i} \mid$ Object) $*$ Prediction (Object) $*$ IOUTruth Prediction = Prediction(Class i) * IOUTruth Prediction

\section{NETWORK DESIGN}

The model is implemented as a convolutional neural network and has been evaluated on the PASCAL VOS detection dataset. The opening layers extract the image features as the output probabilities and coordinates are predicted by the fully connected layers. Image classification taking inspiration from GoogLeNet model is used to develop the network architecture. The network comprises of a total of 24 convolutional layers which are succeeded by 2 fully connected layers. In place of using the inception models by GoogLeNet, simple 1 x 1 reduction layers which are succeeded by 3 x 3 convolutional layers, similar to Lin et $\mathrm{Al}$ are used. An optimized version of YOLO which is mapped out in order to reach the limits of fast object detection is trained. In Fast YOLO, a neural network consisting of fewer convolutional layers (9 instead of 24) and fewer filters are used. All testing and training parameters of YOLO and Fast YOLO are the same other than the size of the network. During the implementation of the model, 1000 images of potholes were used to train the data. Each pothole consisted of details such as height (h), weight (w) and coordinates (x,y) which show the center of the pothole. Using the predictions, the confidence scores of all the potholes were calculated.

\section{TRAINING}

During the implementation of the model, 1000 images of potholes were used to train the data. Each pothole consisted of details such as height (h), weight (w) and coordinates (x,y) which show the center of the pothole. Using the predictions, the confidence scores of all the potholes were calculated.

\section{E. COMPARISON TO OTHER NEURAL NETWORKS}

A primary hurdle in computer vision is object detection. A series of sturdy features from input images are extracted to start detection pipelines. Identification of objects in feature space is done using classifiers and localizers. A comparison is done between YOLO detection system and several top detections frameworks, where the cardinal likeness and disparities are emphasized.

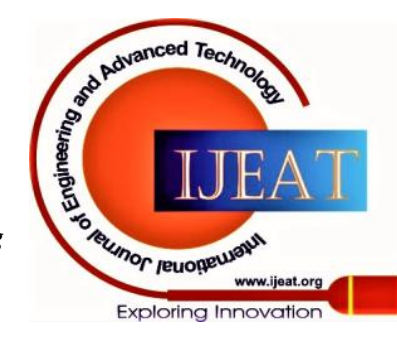



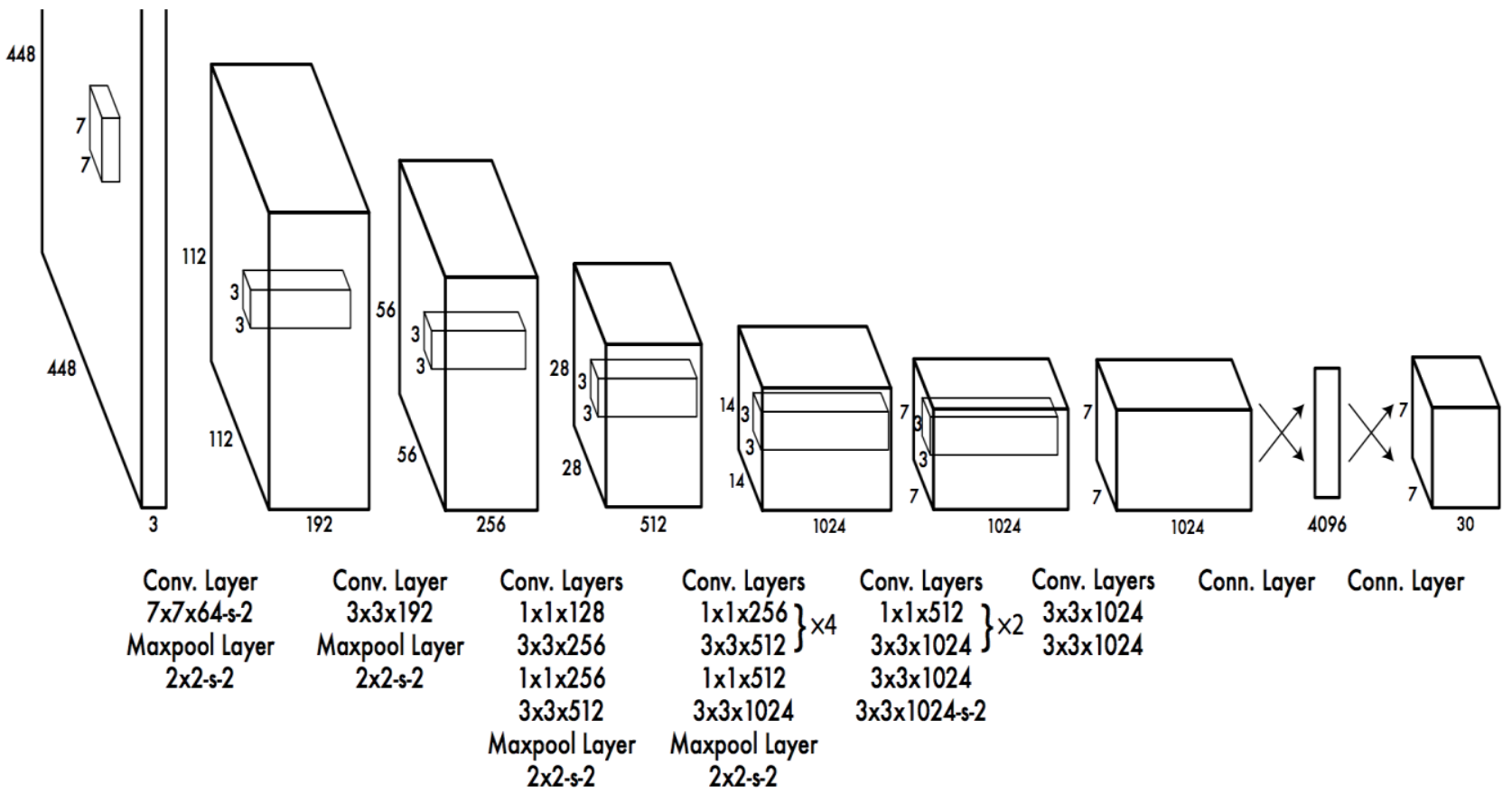

Fig 2: 24 Convolutional Layers and 2 Fully Connected Layers

Deformable Parts Model: Deformable Parts Model, also abbreviated to DPM, makes use of a sliding window approach for object detection. DPM utilizes a disjoint pipeline to extricate static features, classify regions, predict bounding boxes for high scoring regions etc. In place of these contrasting parts, a single convolutional neural network is made available. Making use of contrasting parts is difficult which is why the presence of a single convolutional neural network is a lot more convenient. R-CNN: R-CNN makes use of a different approach to find objects in images, R-CNN and the its varieties utilize region proposals instead of sliding windows. Each stage of this model requires tuning with precision independently and due to this, the resulting system

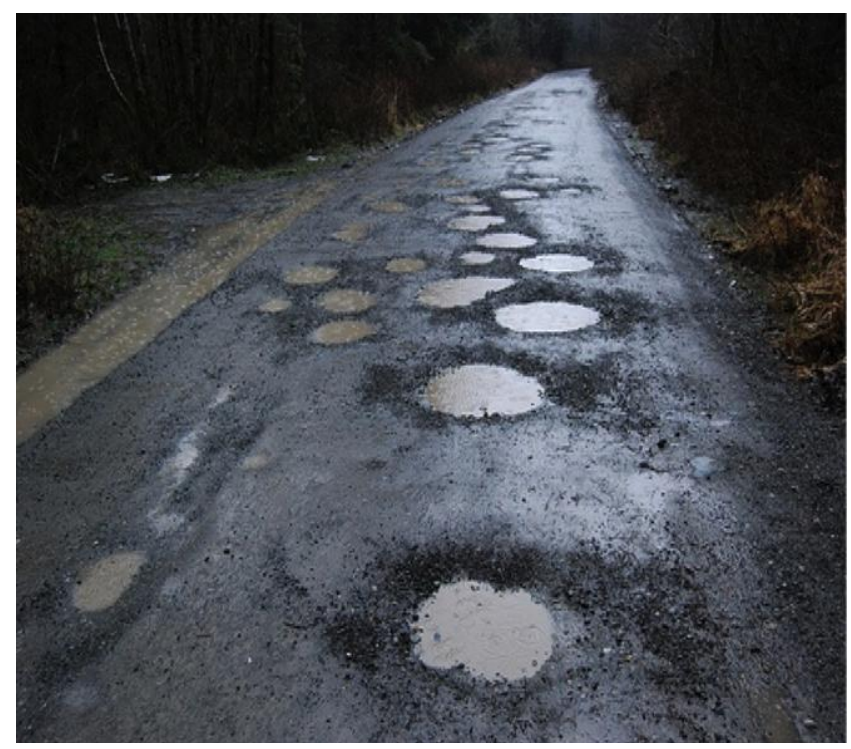

Fig 3: Input Image is very sluggish taking 40 more seconds per image at test time, even though YOLO has some likeness with RCNN.

\section{ANALYSIS AND RESULTS}

Among the different models used throughout the system, that is, YOLO and R-CNN, the following results are found. YOLO is well optimized and performs better when compared to Deformable parts models and R-CNN. YOLO is a widely preferred convolutional neural network model for image classification and object recognition version produced every year. Performing the experiment with the help of TensorFlow as backend, the following results are produced.

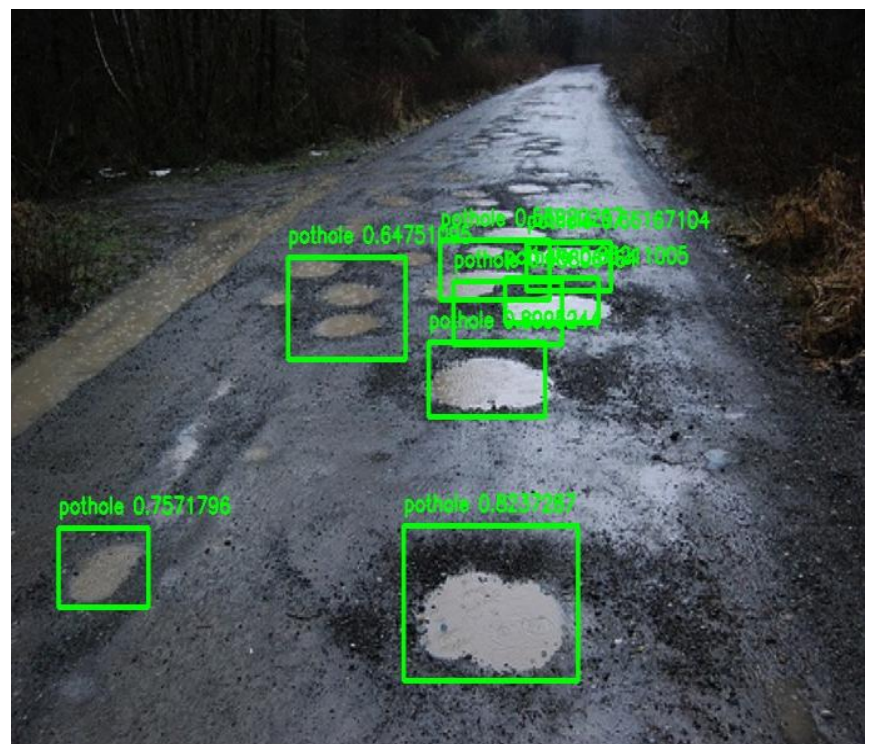

Fig 4: Output/Result

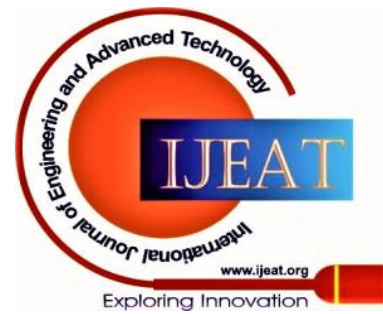




\section{Pothole Detection System}

\section{CONCLUSION}

In conclusion, YOLO, a unified model and convolutional neural network for object detection is implemented. The deep learning model is trained with pothole images to modify and predict potholes. A Deformable parts model or R-CNN model can be trained with the images to predict potholes but comparatively YOLO performs better in predicting potholes and produces promising results in time, speed and accuracy when compared. Better training data could improve the performance model. This model can be packed with flask as an API and integrated with a mobile application to report potholes to the government which will reduce the communication gap between the government and its citizens. This model can also be used in real-time to predict pothole which implemented in autonomous vehicles so that it can prevent or execute a strategy to reduce discomfort to the passengers.

\section{REFERENCES}

1. Artis Mednis, Girts Strazdins, Reinholds Zviedris, Georgijs Kanonirs. Real Time Pothole Detection Using Android Smartphones with Accelerometers. 7th IEEE International Conference on Distributed Computing in Sensor Systems and Workshops (DCOSS) 2011

2. Youngtae Joe, Seungki Ryu. Pothole Detection System Using a Black-box Camera. Highway Research Institute, Korea Institute of Civil Engineering and Building Technology, Sensors. 2015

3. S Gayathri, Mamatha RG, Manasa B , Menita Patil, Sanjana BM. Automatic Pothole Detection

4. System. International Journal of Engineering Research \& Technology (IJERT) 2019

5. Rui Fan, Umar Ozgunalp, Brett Hosking, Ming Liu, Ioannis Pitas. Pothole Detection Based on Disparity Transformation and Road Surface Modeling. IEEE TRANSACTIONS ON IMAGE PROCESSING. Nov 2019

6. S. Gnanapriya, V.B. Padmashree, V. Bagyalakshmi and G.A. Pravallikha. IOT Based Pothole Detection And Notification System. AmericanEurasian Journal of Scientific Research. 2017.

\section{AUTHORS PROFILE}

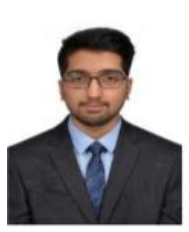

Tushar Premanand- Student at SRMIST, Ramapuram, Chennai pursuing B. Tech in CSE Department, III Year from 2017-2021. Completed high school at Kendriya vidyalaya IIT, Chennai. Mailtusharpremanand@gmail.com

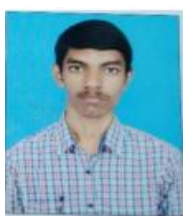

Sriram Sridhar- Student at SRMIST, Ramapuram, Chennai pursuing B. Tech in CSE Department, III Year 2017-2021. Completed High School at Velammal Matric. Hr. Sec School.

Mail- sriramsridhar26@gmail.com

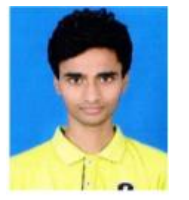

Abhinav Durbha- Student at SRMIST, Ramapuram, Chennai pursuing B. Tech in CSE Department, III Year from 2017-2021. Completed high school at Chinmaya Vidyalaya, Chennai.

Mail- abhinavdurbha@gmail.com 